\title{
PARADIGMAS, DISENOS Y METODOS EN CIENCIA Y TECNOLOGÍA
}

Dr. Gilberto Bustamante Guerrero*

\begin{abstract}
Resumen
Este artículo tiene como objetivo dilucidar, desde una perspectiva epistemológica, el uso de términos cuyo significado ha sido originariamente estipulado en el complejo mundo de la ciencia y de la técnica. Tal es el caso de palabras tales como ciencia, tecnología, teoría, paradigma, diseño, método; términos que tienen como referentes a aspectos importantes de los correspondientes procesos de investigación; pero que, como se puede constatar, son términos que se usan muchas veces siguiendo la moda y en cada caso con significados en los que la vaguedad e imprecisión se suman a la multivocidad que los mismos ya tienen desde su génesis, característica que en el contexto de la diversidad de las escuelas o corrientes metodológicas se torna en un problema complejo y controvertible.
\end{abstract}

Asimismo, tiene el propósito de llamar la atención acerca de la estrecha relación que existe entre el necesario dominio de los elementos sustantivos del paradigma o marco conceptual -entendido a la luz de sus diferentes componentes teóricos, tanto filosóficos como científicos, metódicos, problemáticos, estimativos- y las condiciones de posibilidad de éxito en la tarea de diseñar y desarrollar una investigación científica. Relación importantísima que se replica, mutatis mutandis, en el caso de una investigación tecnológica, cuyas peculiaridades son también puestas de relieve.

Palabras claves:

Metodología, ciencia, tecnología, paradigma, diseño y método.

El examen del lenguaje que empleamos en nuestra vida cotidiana, ya sea en el ámbito familiar, en el trabajo o en cualquier otro escenario social o cultural, nos permite constatar que hacemos uso frecuente de una serie de términos cuyo significado ha sido originariamente estipulado en el complejo mundo de la ciencia y de la técnica; palabras o términos lingüísticos que tienen como referentes a aspectos importantes de los correspondientes procesos de investigación. Éste es el caso de palabras tales como ciencia, tecnología, teoría, paradigma, diseño, método, entre otras. Además, podemos constatar que estos términos se usan muchas veces siguiendo la moda, pero en cada caso con significados en los que la vaguedad e imprecisión se suman a la multivocidad que los mismos ya tienen desde su génesis. Lo señalado no sería particularmente relevante si no fuera porque la vaguedad e imprecisión -que son ciertamente indicadores de la falta de rigor en el uso de dichos términos- no

* Docente de la Unidad de Postgrado de la Facultad de Ciencias Contables. 
ocurriera también en el ámbito del trabajo académico, en campos tan importantes como son los de la investigación científica y tecnológica; $y$, también, en el de la filosofía. Precisamente este hecho es el que motiva la elaboración del presente artículo preparado en el entendimiento que tiene relación con los intereses académicos de quienes se orientan a la investigación científico-social y tienen contacto inmediato con una literatura especializada en la que los aludidos términos y conceptos se usan de tal modo que se justifica el esclarecimiento de los mismos.

Como bien sabemos la filosofía, en una de sus variantes de especialización, orienta sus investigaciones al estudio de la ciencia y de la técnica en lo que respecta a su naturaleza, estructura, lógica, métodos, principios, funciones, criterios de construcción y fundamentación de las teorías y sistemas resultantes, así como de las tensiones a nivel de métodos y teorías rivales, etc. Ello da origen a la denominada Filosofía de la Ciencia o Epistemología, una de cuyas ramas es la Metodología de la Ciencia, disciplina a la que me referiré, en primer término, con el propósito de identificar su objeto de estudio y sus funciones, al mismo tiempo que comprender algunos aspectos de la controversia que subyace en su desarrollo histórico, elementos necesarios como marco de referencia a las consideraciones específicas que formularé en relación con los temas materia del presente trabajo.

\section{LA METODOLOGÍA DE LA CIENCIA}

La problemática filosófica en torno a la ciencia se caracteriza por ser compleja, diversa y controvertible. En tal contexto, un tipo de problema que algunos pensadores consideran relevante es el referido al método de investigación científica tomado como objeto de estudio teórico-filosófico. Se trata propiamente de un área problemática al interior de la cual es posible identificar numerosos y novedosos problemas específicos. Para este efecto, a mi juicio, es necesario en primer lugar partir de un marco conceptual que ofrezca una clara definición de elementos constitutivos, propiedades, características y relaciones para enseguida, lograr la identificación de temas cuya importancia, autenticidad $y$ originalidad los conviertan en problemas de investigación cuyos resultados sean de interés y utilidad para científicos y metodólogos, especialistas estos últimos que participan tanto en la investigación científica cuanto en la formación de investigadores.

En general, se reconoce que a la Metodología de la Ciencia le corresponde, por lo menos desde cierta perspectiva, la tarea de cuestionar radical y reflexivamente los aspectos metódicos de la investigación científica. Sabemos que etimológicamente es entendida como teoría del método e históricamente se ha constituido en una rama importante de la Filosofía de la Ciencia.

Particularmente, Karl Popper afirma que la Epistemología -o la lógica de la investigación científica, como él suele denominarla-debería identificarse con la teoría del método científico en cuanto ésta se ocupa de la elección de las decisiones acerca del modo de habérselas con los enunciados científicos en relación muy estrecha con el criterio de demarcación. Ahora bien, si entendemos como método científico, en términos genéricos, a la estrategia o método general de la investigación, ya sea natural o social, pura o aplicada, formal o fáctica, estamos pues refiriéndonos a un proceso o curso de acción, constituido por un conjunto o ciclo de pasos $\mathbf{u}$ operaciones, cada uno de los cuales es realizado por los 
científicos teniendo en cuenta la particular naturaleza de su objeto de estudio $y$ mediante específicos procedimientos técnicos expresables en reglas, que en este caso se conocen como las reglas del método científico.

Los epistemólogos, en primer término, se proponen un objetivo descriptivo, el cual implica ya serias dificultades, sobre todo si no son también investigadores científicos; $y$, logrado éste, pretenden alcanzar un objetivo normativo, de suerte que el epistemólogo, en tanto metodólogo, resulta erigiéndose en orientador y evaluador de la investigación científica y de sus resultados, las teorías científicas.

La metodología en su función descriptiva del método científico consiste principalmente en la reconstrucción racional de los procesos de operación de los científicos en cuanto investigadores, los criterios de elección entre procedimientos específicos y cursos de acción en competencia, la elección, uso y función de modelos, el uso de diseños experimentales, los objetivos y formas de la predicción científica, los criterios de justificación de las reglas del método, así como los criterios para la construcción de teorías científicas. Los logros en este campo han sido significativos; sin embargo, debemos recordar que asumir el concepto de método general de la ciencia implica ya una decisión discutible y que las reconstrucciones racionales logradas corresponden a investigaciones en algunos campos de ciencias muy conocidos y más bien tradicionales, habida cuenta la vertiginosa profundización y diversificación del conocimiento científico.

Por otro lado, la metodología de la ciencia en su función normativa, que como dijimos líneas arriba, pretende ser directora del proceso de investigación, cumple su tarea a partir de las limitaciones de los incompletos estudios descriptivos y, estima, que solamente resulta de interés y utilidad para los científicos cuando alcanza a formular un sistema de reglas que no solamente ayudan a evitar errores en la investigación científica sino que la mera justificación pragmática que hasta ahora tienen, en tanto son útiles por sus resultados, alcancen a integrar un sistema de reglas fundamentadas teoréticamente, en la medida en que tal sistema de reglas esté basado y sea compatible con las leyes de la lógica y las leyes de la ciencia.

Además, recordemos que la metodología, como rama de la epistemología, es un quehacer filosófico que necesita del acercamiento imprescindible a la experiencia científica en todo el proceso de investigación y producción y que sólo será capaz de sugerir nuevos enfoques promisorios en la investigación si los metodólogos, además de filósofos, tienen suficiente formación y experiencia en la investigación científica. Recordemos también que, en un sentido, la historiografía de la ciencia nos permite conocer que las reglas del método son falibles y requieren perfeccionamiento; que, si bien ayudan a evitar errores, no aseguran originalidad; que la secuenciación de reglas producto del trabajo de reconstrucción racional que realizan los metodólogos es tan sólo un arreglo ya que en la práctica de la investigación los pasos orientados por reglas interactúan constantemente. Asimismo, en otro sentido y en términos kuhnianos, las normas metódicas que se emplean en un momento dado son tan sólo elementos integrantes de un «paradigma», entendido como el "conjunto de valores compartidos» (métodos, nociones, generalizaciones) por los investigadores en un momento de 
"ciencia normal»; y, entendido también como «realización», es decir como forma acertada para resolver un problema que entonces sirve como modelo para futuros investigadores.

Finalmente, es del caso señalar enfáticamente que la variedad de escuelas, corrientes y planteamientos que caracterizan la controversia filosófica en torno a la ciencia se reproduce en la rama metodológica. Más aún, Bunge no solamente afirma que hay varias metodologías a flote en la actual filosofía de la ciencia sino que "metodología" actualmente $-y$ a diferencia de lo que se pensaba en el siglo XVIII- ya no se entiende como un «libro de reglas fijas para resolver problemas" sino tan solamente como "un conjunto de reglas (acaso ni siquiera estrechamente entretejidas, ya no digamos mecánicas) para la evaluación de teorías ya formadas y articuladas", añadiendo que a menudo estas reglas, o sistemas de evaluación, también sirven como «teorías de racionalidad científica», "normas de demarcación» de "definiciones de la ciencia", citando como ejemplo cuatro distintas metodologías: Inductivismo, convencionalismo, falsacionismo metodológico y metodología de los programas de investigación científica.

Ahora bien, al establecer nuestro marco de referencia, hemos ubicado los conceptos de paradigma y método; términos que, conjuntamente con el de diseño, reclaman un necesario esclarecimiento, no solamente por su importancia -derivada de su estrecha relación con el proceso de investigación científica- sino porque vienen siendo utilizados en los tratados de metodología de manera tal que generan confusión. Por las razones expuestas, en lo que sigue intentaré esclarecer el sentido en el que considero que debieran ser utilizados dichos términos.

\section{PARADIGMAS}

En los últimos cuarenta años el término 'paradigma' ha sido usado de modo intensivo a partir del momento en que Thomas Kuhn publica su notable obra titulada La estructura de las revoluciones científicas (1962). En este trabajo su autor define "paradigma", según mencioné hace un momento, como "conjunto de valores compartidos» $\mathrm{y}$ también como «realización». El mismo Kuhn en el prefacio de su libro, refiriéndose a los "paradigmas" afirma: "Considero a éstos como realizaciones científicas universalmente reconocidas que, durante cierto tiempo, proporcionan modelos de problemas y soluciones a la comunidad científica».

Mario Bunge, en su interesante libro Seudociencia e ideología (1985), reconoce el mérito de Kuhn en el sentido de llamar la atención sobre las características de las tensiones conceptuales, factores y cambios radicales a los que llama "revoluciones científicas", las que ocurren en el proceso histórico de avance del conocimiento. Además, considera que el significado de "paradigma" en Kuhn es compatible con la expresión «estilos de pensamiento» utilizada por Ludwig Fleck en su libro Génesis y desarrollo del hecho científico (1935) y ambas expresiones a su vez compatibles con el significado de "marco conceptual", al que caracteriza como un campo epistémico que cuando asume un rol dominante con respecto a otros de su tipo se le denomina paragón. Sin embargo, Bunge piensa que lo que sigue siendo problema son las nociones mismas de marco conceptual, de paradigma y de revolución en el paradigma, en la medida en que ninguna de estas nociones ha sido dilucidada por Kuhn ni por quienes como Imre Lakatos y Paul Feyerabend se presentan como sus seguidores. Éstos, y otros, son algunos términos del debate filosófico, que 
solamente menciono de paso y de manera inevitablemente incompleta, porque creo que por es preferible no eludir su mención, siempre de interés cultural; aún cuando, por diversas razones y condiciones, no es el caso el involucrarnos en especializados temas filosóficos, los que por ser sustancialmente controvertibles son objeto de un debate que no tiene solución de continuidad.

En consecuencia, me parece muy razonable y de efectos esclarecedores $-y$ en este sentido va mi propuesta- asumir el significado de 'paradigma' definiéndolo por extensión, con los mismos elementos constitutivos con los cuales Bunge define aquello que denomina «marco conceptual». Su comprensión y manejo por profesionales y estudiantes que tienen interés en cualificar sus tareas intelectuales como investigadores en ciencia y tecnología se verán enriquecidos con esta aproximación conceptual, por cuanto de este modo nuestras consideraciones estarán formuladas con ideas más claras en relación con las dificultades inherentes a las tareas implícitas a todo proyecto de investigación, tal como el formular o elegir, según el caso, los diseños, problemas, marco teórico, etc.

Comenzaré por señalar una situación muy conocida, como es el caso de los componentes de la cultura contemporánea, en la que existen y tienen sus propias particularidades como órdenes o esferas distinguibles el arte, ideología, tecnología, humanidades, filosofía, ciencia, matemática. En su conjunto comparten la condición de ser campos de conocimiento en los que de acuerdo a los criterios para su constitución, fundamentación y desarrollo, así como a su velocidad de cambio se puede reconocer y distinguir, por un lado, a los llamados campos de creencias entre las que cuentan las ideologías totales, religiones, ideologías políticas, seudociencias y seudotecnologías; $y$, por otro, a los llamados campos de investigación, entre los que identificamos a las humanidades, las ciencias formales, las ciencias básicas y las aplicadas, así como a todos los tipos de tecnologías.

Un campo de investigación, según nuestro autor, está compuesto de un marco material y un marco conceptual. El primero conformado por una comunidad de investigadores, la sociedad que apoya la investigación y los hechos de la realidad constituidos como objeto de estudio. El segundo, es decir el marco conceptual que nos interesa en especial porque su comprensión nos lleva a la comprensión del concepto de paradigma a su vez está constituido por un complejo conjunto de elementos de naturaleza espistémica entre los que identifica a los siguientes:

1. Una concepción general o transfondo filosófico, integrado por axiomas o principios filosóficos, especialmente en lo que respecta a una ontología de los hechos, una gnoseología realista que incluya la noción de verdad, una ética en la investigación para alcanzar la verdad objetiva.

2. Una colección de teorías y métodos lógicos y matemáticos, en particular algoritmos; constituyen un transfondo formal.

3. Un cuerpo de conocimientos integrado por una colección de datos, hipótesis, teorías y métodos bien confirmados y actualizados, todos ellos tomados en préstamo de otros campos de investigación; integran un transfondo específico.

4. Una colección de problemas que pueden ser investigados constituyen la problemática que tiene la 
particularidad de ser exclusiva con respecto a sus referentes, que son los hechos o entes reales que constituyen un área reconocible como dominio o universo del discurso.

5. Un fondo de conocimientos acumulados -o colección de datos, hipótesis, teorías y métodosobtenidos previamente por miembros de la comunidad de investigadores del dominio, y que son compatibles con el cuerpo de conocimientos que conforman el transfondo específico.

6. Objetivos o metas de la investigación; entre los que se incluye el de formular hipótesis y asentar leyes acerca de los hechos que pertenecen al dominio del discurso, estructurar teorías y afinar los métodos de investigación.

7. Una metódica o colección de métodos para usar en la investigación; métodos cuya justificación pragmática debe ser cuidadosamente evaluada.

Un investigador científico para cumplir su tarea, como ocurre regularmente y es esperable, debe diseñar un proyecto de investigación. Es una tarea medular cuya realización requiere de una formación especializada que implica el conocimiento y práctica suficientes con respecto a todos y cada uno de los componentes del marco conceptual o paradigma pertinente. Una manera muy recomendable para comenzar a diseñar y desarrollar proyectos de investigación, además de haber estudiado los métodos y teorías del respectivo campo de investigación, consiste en elegir como modelo a una investigación ya realizada exitosamente. Esta investigación es denominada ejemplar y en tanto proporciona a una comunidad científica un modelo de problema y de su respectiva solución es, por cierto, una manera eficiente para aplicar un paradigma vigente y acaso dominante en la esfera específica de una de las ciencias.
Sin embargo, es sumamente importante señalar que las ciencias y tecnologías tienen desarrollos desiguales; que algunas son muy jóvenes, como las ciencias sociales, y que por su condición de ciencias emergentes o en formación no tienen claramente desarrollados aquellos elementos de naturaleza epistémica que constituyen el marco conceptual, y que propiamente aún no es posible hablar de paradigmas ya logrados, sino precisamente también en formación.

\section{DISEÑOS $Y$ MÉTODOS DE INVESTIGACIÓN}

Acabamos de concluir que la condición de éxito en la tarea de diseñar un proyecto de investigación pasa por conocer -teóricamente, prácticamente y a muy buen nivel- los elementos componentes del respectivo marco conceptual, aún cuando no esté claramente logrado. Es necesario, pues, aprehender el correspondiente «estilo de pensamiento". Sin cumplir este requisito la aventura intelectual de investigar genuinamente en ciencia, y también en tecnología, es inviable.

En los tratados y manuales que contienen reglas, normas, criterios y consejos para realizar una investigación uno de los temas es el relacionado con los diseños de investigación. Y como se puede comprobar fácilmente al respecto, en dichos tratados y manuales se habla de diseños de investigación en sentidos distintos y a veces con mucha superficialidad. Ocurre que una de las causas de tal diversidad y confusión deriva de utilizar la expresión para hablar de distintos referentes, los que -a su vezson identificados de acuerdo a la visión del proceso de investigación, ya sea desde una perspectiva general $o$, puntualmente, en referencia a alguna etapa o acción específica del aludido proceso. 
En todo caso, muchas veces el resultado no solamente es desconcertante sino incluso frustrante para quienes se inician en la tan importante tarea de investigar, como es el caso de quienes preparan una tesis de grado o de postgrado. En realidad, una de las causas es la carencia de una razonable formación filosóficoepistemológica y científica, inclusive en un número significativo de los propios autores de manuales, quienes salvo contadas excepciones, se presentan muy orondos como metodólogos y no pasan, muchas veces, de ser simples compiladores de trabajos que, además, no articulan bien.

No pretendo en este trabajo otra cosa que llamar la atención acerca de la estrecha relación que existe entre el suficiente conocimiento del paradigma o marco conceptual y las condiciones de posibilidad de éxito en la tarea de diseñar un proyecto de investigación. En otras palabras: Quien no tiene conocimientos epistemológicos acerca del proceso y lógica de la ciencia, y tampoco tiene dominio de los conceptos y teorías científicas exitosas en relación con cierto sector del universo fáctico, ni acerca de la problemática y metódica correspondientes, no está ciertamente en condiciones de realizar un tipo de trabajo que requiere de racionalidad, objetividad y eficiencia, a lo largo del proceso que va desde la identificación de un problema de investigación hasta la valoración de sus resultados en el cuerpo de conocimientos disponibles.

Sin embargo, no quisiera dejar pasar la ocasión sin mencionar algunos comentarios y apreciaciones. En principio, creo que es sugerente y una aceptable aproximación la definición que Fred Kerlinger en su libro Investigación del Comportamiento. Técnicas y metodología, para quien "Diseño es el plan, estructura y estrategia de una investigación cuyo objetivo es dar respuesta a ciertas preguntas y controlar la varianza», especificando lo siguiente:

a. Que, diseño como plan es un esquema global o programa en el que bosqueja todo lo que realizará el investigador;

b. Que, diseño como estructura es el bosquejo específico de operación de las variables; $y$,

c. Que, diseño como estrategia es la previsión y determinación de los métodos para reunir y analizar datos en atención a los objetivos de la investigación.

Como se puede apreciar, diseño se usa en tres sentidos diferentes pero no excluyentes sino complementarios, dado su nivel de generalidad o especificidad. Sin embargo, como son muy pocas las obras de metodología en los que se precisa el sentido en el que se usa en cada caso, la confusión es una consecuencia no solamente previsible sino frecuente en la práctica.

Elaborar el diseño de un proyecto de investigación, en el sentido de plan, a su vez también puede dar lugar distinguir los aspectos teórico-metodológicos de aquellos aspectos de presupuesto, financiamiento, logística, etc., situación que permite hablar, con propiedad, de un "diseño académico" y de un "diseño administrativo». El primero de estos diseños tiene un prerrequisito ineludible: Una muy clara comprensión de la lógica de la investigación, por cuanto el plan de trabajo debe atender secuencial y muy rigurosamente a todas y cada una de las etapas $u$ operaciones relevantes de las que consta el ciclo completo de la investigación, cuyo desarrollo está regulado o prescrito por las normas, reglas y criterios del método general de la ciencia. Estas consideraciones demuestran el estrecho vínculo lógico que existe entre el diseño de investigación como plan y el método general de la ciencia. 
El diseño de investigación entendido como estructura, en un caso, o como estrategia, en otro, a su vez da lugar a una muy nutrida serie de desarrollos metodológicos, cuya taxonomía sería realmente muy interesante, ilustrativa y útil; desarrollos que son explicables por cuanto los bosquejos de esquemas de relación $\mathbf{u}$ operación entre variables son muy numerosos, como numerosos son también los instrumentos específicos y los criterios y técnicas, para cumplir una a una todas las operaciones que permitan garantizar el logro de los objetivos de la investigación, con la suficiente racionalidad y objetividad, distintivos del pensamiento científico.

En relación con el proceso de investigación tecnológica, existe una problemática epistemológica escasamente desarrollada pero que en las últimas décadas al parecer muestra un cierto cambio que no quisiera dejar de mencionar, por lo menos en términos referenciales, por cuanto estudios recientes revelan la existencia de métodos y criterios de investigación que, obviamente, responden a marcos conceptuales o paradigmáticos y también se relacionan con los diseños de los correspondientes proyectos de investigación tecnológica. El tema es muy amplio, pero me interesa poner de relieve algunas ideas.

En efecto, pienso que es el caso llamar la atención acerca de un hecho: el desinterés epistemológico por estudiar el método de la tecnología es una tendencia que ocurre, precisamente, en sentido contrario al del proceso real de investigación en el mundo desarrollado, en el que el mayor interés, inversión y producción de conocimientos corresponde a la investigación tecnológica; más aún, tal tendencia no sería casual, si observamos que la asimetría es totalmente compatible y coincidente con la política dominante de difusión de los resultados de la investigación, que es relativamente abundante en información científica y en extremo restringida en información tecnológica. En este sentido, la filosofía estaría reflejando los efectos de una política de dominación en favor de quienes, a partir de una injusta división internacional del trabajo, reservaron para sí el rol de productores de ciencia y tecnología.

La situación que hemos reseñado tiene una expresión constatable: La confusión conceptual sobre la naturaleza de la tecnología, de sus dimensiones, componentes, método de investigación, ramas, ubicación en el contexto de la cultura contemporánea; y, especialmente, el escaso y muy confuso conocimiento de su relación con la ciencia. La confusión descrita constituye una dificultad importante que afrontan no solamente los filósofos sino también los investigadores tecnólogos y los tecnólogos en acción. Esta dificultad, además, se incrementa en los países tecnológicamente dependientes, debido a la carencia de procesos reales de investigación tecnológica y a la escasa disponibilidad de información sobre los resultados de las investigaciones científicas y tecnológicas, especialmente de estas últimas, realizadas en países desarrollados.

Felizmente la situación que acabamos de describir tiende a cambiar. Se observa en los países desarrollados un incremento del interés filosófico por la técnica científica moderna, cuya problemática adquiere relevancia -debido a que su notable desarrollo e impacto en la vida individual y social plantea la necesidad de disponer de respuestas críticas y sistemáticas a importantes cuestiones- $y$ genera el desarrollo todavía incipiente de la hoy llamada Filosofía de la Tecnología. La problemática propia de esta Filosofía de la Tecnología no pasa de ser, todavía, 
un interesante programa de trabajo, complejo, diverso y no exento de controversias pero de grandes proyecciones, por cuanto el aludido incremento del interés filosófico por la tecnología abarca actualmente una mayor amplitud temática, que supera la inicialmente abordada, en tanto no se limita a examinar aspectos externos de la tecnología, analizando ética y antropológicamente el impacto de ésta en la vida individual y social, sino que abre nuevas áreas de exploración ontológica, axiológica, política, lógica, metodológica.

El señalado desarrollo de los estudios filosóficos en torno a la tecnología resulta, pues, una tendencia creciente al interior de la cual creo que resultan de gran importancia los estudios metodológicos, porque su realización obliga al metodólogo a una permanente aproximación al proceso real de la tecnología misma. De este modo, no solamente genera la posibilidad de que investigue genuinos problemas filosóficos acerca de la metódica de la investigación tecnológica -distinguibles por su autenticidad, originalidad y envergadura- sino porque también apertura la posibilidad de que sean los investigadores tecnólogos los que conozcan los resultados de las investigaciones metodológicas, que se espera consistan en soluciones claras a los problemas estudiados, en criterios adecuados para distinguir tecnología de pseudotecnología, en criterios de evaluación y en planteamientos de crítica y corrección de sistemas tecnológicos.

De lo que acabamos de mencionar se deriva que la problemática de la Metodología de la Tecnología es sin duda alguna bastante amplia. Sin embargo, una de sus tareas primordiales consiste en la delimitación y ubicación de su casi inexplorado objeto de estudio: la metódica de la investigación tecnológica.
Estimo que esta tarea puede ser abordada mediante una aproximación al marco contextual de la metódica de la investigación tecnológica: el proceso real de investigación y desarrollo científicotecnológico, en el marco, también real, de la sociedad concreta. Pienso que tal aproximación nos permitirá identificar algunas imprecisiones de orden conceptual e intentar su esclarecimiento.

A partir del análisis de las etapas del proceso real de investigación $y$ desarrollo, queda identificada una etapa -de investigación tecnológica- en la que se reconoce una secuencia metódica propiamente tecnológica, en razón de sus objetivos, pasos y criterios. A este respecto destaca precisamente la ubicación de las operaciones de formulación y contrastación empírica de la predicción tecnológica como operaciones que forman parte de los pasos de diseño y prueba. Ahora bien, decimos que esta secuencia metódica es propiamente tecnológica porque en ella se reconocen peculiaridades tácticas, que he identificado en un trabajo que preparé hace algunos años, y que se expresan en términos de: la técnica específica para discernir el problema tecnológico de naturaleza práctica; las alternativas de solución, que se formulan en función de los parámetros eficiente-ineficiente, inherentes al campo del conocimiento para la acción; el nivel de concreción de sus puntos de partida, los elementos que se someten a contrastación y los objetivos de ésta.

No cabe duda, pues, que este enfoque aproximativo a la realidad del proceso de investigación y desarrollo resulta fecundo, de modo tal que dada su utilidad debemos tenerlo muy en cuenta para aplicarlo si fuere pertinente, aún cuando es obvio que muchas y muy importantes cuestiones quedan sin ser mencionadas. 


\section{RESUMEN}

A partir del análisis conceptual, lógico y lingüístico propios de la Epistemología o Filosofía de la Ciencia es posible constatar que no solamente en el ámbito del habla coloquial sino también en el mundo académico hacemos uso frecuente de términos tales como paradigma, diseño, método, metodología, teoría, ciencia, tecnología, etc., los mismos que se usan sin el rigor que demanda su multivocidad $y$, peor aún, con significados en los que la vaguedad e imprecisión generan confusión conceptual, grave defecto que afecta sustantivamente los niveles del pensamiento y de la acción. Este hecho justifica la necesidad de esclarecer los conceptos básicos de la ciencia y la tecnología desde una perspectiva epistémica como un modo de mejorar la calidad de la formación profesional universitaria y de los procesos de investigación.

La comprensión del concepto de paradigma científico o tecnológico, en función de los elementos constitutivos del respectivo marco conceptual, es particularmente importante para pensar y actuar con propiedad en un campo de investigación. En este respecto, la condición sine qua non de éxito en la tarea de diseñar un proyecto de investigación y desarrollarlo, pasa por conocer -teóricamente, prácticamente y a muy buen nivel- los elementos componentes del respectivo marco conceptual o estilo de pensamiento, aún cuando éste, en algunas ciencias o tecnologías por su condición de emergentes, no haya sido claramente desarrollado. Sin cumplir este requisito la aventura intelectual de investigar genuinamente en ciencia, y también en tecnología, es inviable.

\section{BIBLIOGRAFÍA}

POPPER, Karl R. La lógica de la investigación científica. Madrid, Editorial Tecnos S.A., 1962.

KUHN, Thomas S. La estructura de la revoluciones científicas. México, Fondo de Cultura Económica, 1980.

- $\quad$ LAKATOS, Imre. Matemáticas, ciencia y epistemología. Madrid, Alianza Editorial S.A., 1981.

- FEYERABEND, Paul. Tratado contra el método. Madrid, Editorial Tecnos, S.A., 1981.

BUNGE, Mario. Epistemología. Barcelona, Editorial Ariel, 1980.

- BUNGE, Mario. Seudociencia e ideología. Madrid, Alianza Editorial, 1985.

- KERLINGER, Fred. Investigación del comportamiento. Técnicas y metodología. México, Interamericana, 1979. 